\author{
Jean-Blaise Wasserfallen \\ Jean-Pierre Revelly \\ Davide Moro \\ Nicolas Gilliard \\ Jacques Rouge \\ René Chioléro
}

\section{Can the impact of bed closure in intensive care units be reliably monitored?}

Received: 21 January 2003

Accepted: 27 January 2004

Published online: 28 February 2004

(C) Springer-Verlag 2004

\section{J.-B. Wasserfallen $(\bullet)$}

Medical direction, CHUV-BH 08,

Rue du Bugnon, 1011 Lausanne,

Switzerland

e-mail: jwasserf@ chuv.hospvd.ch

Tel.: +41-21-3141802

Fax: +41-21-3141818

J.-P. Revelly $\cdot$ J. Rouge $\cdot$ R. Chioléro

Surgical Intensive care unit,

University Hospital,

Lausanne, Switzerland

D. Moro

Department of Surgery,

University Hospital,

Lausanne, Switzerland

N. Gilliard

Service of Anesthesiology,

University Hospital,

Lausanne, Switzerland

\begin{abstract}
Objective: To assess the properties of various indicators aimed at monitoring the impact on the activity and patient outcome of a bed closure in a surgical intensive care unit (ICU). Design: Comparison before and after the intervention.

Setting: A surgical ICU at a university hospital. Patients: All patients admitted to the unit over two periods of 10 months. Intervention: Closure of one bed out of 17. Measurements and results: Activity and outcome indicators in the ICU and the structures upstream from it (emergency department, operative theater, recovery room) and downstream from it (intermediate care units). After the bed closure, the monthly medians of admitted patients and ICU hospital days increased from 107 (interquartile range 94-112) to 113 (106-121, $P=0.07)$ and from $360(325-443)$ to 395 (345-436, $P=0.48$ ), respectively, along with the linear trend observed
\end{abstract}

in our institution. All indicators of workload, patient severity, and outcome remained stable except for SAPS II score, emergency admissions, and ICU readmissions, which increased not only transiently but also on a mid-term basis (10 months), indicating that the process of patient care delivery was no longer predictable. Conclusions: Health care systems, including ICUs, are extraordinary flexible, and can adapt to multiple external constraints without altering commonly used activity and outcome indicators. It is therefore necessary to set up multiple indicators to be able to reliably monitor the impact of external interventions and intervene rapidly when the system is no longer under control.

Keywords Intensive care · Efficiency · Quality indicators · Hospital bed capacity $\cdot$ Health care rationing $\cdot$ Economics

\section{Introduction}

The search for medical and economic efficiency criteria to close beds to eliminate excess hospital capacity and reduce costs is of importance [1]. Such measures can overshoot and lead to threats to patient flow and quality of care, particularly during periods of peak activity and in case of emergency admissions. To defend allocation decisions for finite resources, such as hospital beds, two different modeling techniques have been used [2]: the bed occupancy management and planning system (BOMPS), and Sorensen's multi-phased bed modeling
(MPBM). In addition, simulation models have shown that the risk of bed shortages for accommodating emergency admissions exists when average bed occupancy rate exceeds $85 \%$ [3]. Even for standardized care such as cardiovascular surgery, after which $89.5 \%$ of patients experience an intensive care unit (ICU) length of stay (LOS) shorter or equal to $48 \mathrm{~h}$, considerable overall variation exists [4]. The distribution of LOS has a long tail. LOS requires a high degree of resource capacity to avoid operation cancellations because of unavailability of suitable postoperative care [5]. It is thus important to monitor the impact of interventions aimed at reducing 
hospital bed numbers to adapt these measures to an optimum.

Intensive care beds are especially attractive in this context, as ICUs are high-technology, effective but increasingly expensive units. Closure of one bed saves several employees positions [6, 7]. However, ICU resource availability is essential for many other activities and services in a hospital. This is particularly relevant in a surgical department managing patients with severe trauma, major surgery, or organ transplantation. This also affects the regulation of patient flow between ICU, intermediate care units, and recovery room. Therefore, ICU size must be correctly tailored to existing needs to prevent serious and potentially adverse impacts on the way the whole hospital functions.

Several indicators are available to monitor ICU patients $[8,9,10,11,12]$, but their value and responsiveness to assess the impact of restrictions in access to ICU have never been studied. Quality of care indicators usually focus on structure, processes, and outcome measures [13]. We selected a panel of routinely collected indicators in these three domains to assess their response to the closure of one ICU bed for economic reasons at our institution.

\section{Methods}

The study was performed in an 850-bed university hospital, serving as city hospital for Lausanne (250,000 inhabitants), a secondary care center for a population of 650,000 inhabitants, and one of the two university hospitals in French-speaking Switzerland (1.8 million inhabitants). The surgical department has a surgical ICU of 17 beds, and five subspecialty intermediate care units totaling 31 beds [cardiosurgery (six beds), general surgery (12 beds), neurosurgery (five beds), ENT surgery (four beds), and orthopedic surgery (four beds)]. The surgical ICU operates as a "closed unit". Decisions regarding admissions and transfers are under the responsibility of the attending physician of the ICU, in collaboration with the surgeon in charge of the patient, according to written and established criteria for both admission to ICU and transfer to intermediate care units. These intermediate care units are operated by the surgical staff only.

Structure, process, and outcome indicators within and outside the ICU were monitored over a 10-month period before (January 1997 to October 1997) and after (November 1997 to August 1998) the closure of one bed out of 17 in our surgical ICU (6\% decrease in capacity). All data were routinely collected and therefore available. These included administrative data (age, gender, diagnoses, operations), number of admissions and ICU days, both for elective and emergency cases, and occupancy rate, computed as the ratio between the highest number of patients present in the unit at any time in the 24-h period divided by the number of available beds. Patient-related indicators extracted from the medical chart included severity scores (Simplified Acute Physiology Score SAPS II at $24 \mathrm{~h}$ for ICU patients [9], American Society of Anesthesiology score (ASA) [10] at premedication for recovery room patients), and daily nurses workload (Patient Research Nursing (PRN) [11]). The PRN system compares the workload provided in the care unit by the staff present during a given day (provided workload) to the workload that the patient's severity of disease would theoretically need (requested workload). One point is equal to 5 min of nursing work.
In the ICU, the patients were classified into four categories, according to the criteria of the Swiss Intensive Care Society (Société Suisse de Médecine Intensive (SSMI) [12]), and based on the required nurse per patient ratio per shift: category IA: $>1$ nurse per patient per shift; IB: one nurse per patient per shift; II: one nurse per two patients per shift; III: one nurse per three patients per shift. This system has been used for more than 20 years in Swiss ICUs [12].

Outcome indicators included mortality and readmissions to take into account early discharges. Readmission was defined as a second ICU admission for the same medical condition occurring within 4 days of ICU discharge. A second ICU admission for the same medical condition later after discharge or for a different medical condition was considered a new admission. External indicators included patient ward destination after emergency room admission and recovery room stay (reflecting impact on upstream units), as well as number of admissions and nursing workload in intermediate care units (reflecting impact on downstream units). During the study time-period, no change in technology, process of care, physician coverage, structure or staffing of other hospital units occurred, as documented by administrative and accounting data.

Comparisons were carried out with Mann Whitney U-test (correcting for multiple tests) or chi-square test when appropriate, and relationships between the different variables were assessed by Spearman coefficient correlation. All analyses were carried out on SPSS, version 10.0. Statistical significance was assumed at $P<0.05$. As this study involved assessment of processes of care and not patients directly, submission of the protocol to the ethics committee of our institution was not required.

\section{Results}

\section{Hospital activity}

During the years 1997 and 1998, 22,709 and 39,491 patients were admitted, respectively, representing 218,882 and 367,398 hospital days, respectively. In the same years, 16,170 and 16,642 surgical operations were carried out, respectively.

\section{ICU activity and patient outcome}

After bed closure, the monthly medians of admitted patients and ICU hospital days increased from 107 (interquartile range 94-112) to $113(106-121, P=0.07)$ and from $360(325-443)$ to $395(345-436, P=0.48)$, respectively. The results of available indicators for ICU activity and patient outcome before and after bed closure are displayed in Table 1. Altogether, the number of admissions increased after bed closure, mainly due to a statistically significant increase in the number of emergency admissions. This led to an increase in the number of hospital days which was statistically significant for the proportion of hospital days dedicated to emergency admissions. No reproducible seasonal pattern was observed during both observation periods (Fig. 1), but the rate of emergency admissions was more closely correlated to the number of admissions after bed closure than before 
Table 1 Comparison of ICU activity and outcome indicators before and after bed closure (median of monthly distributions)

\begin{tabular}{lclclc}
\hline & Before & (Quartiles) & After & (Quartiles) & $\begin{array}{l}\text { Statistical } \\
\text { significance }\end{array}$ \\
\hline Admissions number & & & & & \\
$\quad$ Total & 107 & $(94 ; 112)$ & 113 & $(106 ; 121)$ & 0.07 \\
$\quad \begin{array}{l}\text { Emergency (\%) } \\
\text { Hospital days number }\end{array}$ & 47 & $(41 ; 49)$ & 56.4 & $(46.7 ; 61.0)$ & 0.02 \\
$\quad$ Total & 360 & $(325 ; 443)$ & 395 & $(345 ; 436)$ & 0.48 \\
Emergency (\%) & 66.0 & $(63.7 ; 69.0)$ & 70.6 & $(67.9 ; 74.0)$ & 0.04 \\
Occupancy rate (\%) & 69.1 & $(63.4 ; 86.1)$ & 79.7 & $(70.8 ; 88.6)$ & 0.22 \\
Intensive care categories (\%) & & & & & \\
Cat. 1a & 6.3 & $(4.5 ; 10.4)$ & 6.7 & $(5.9 ; 10.6)$ & 0.32 \\
Cat. 1b & 73.3 & $(70.5 ; 76.9)$ & 66.9 & $(63.0 ; 70.7)$ & \\
Cat. 2 & 17.1 & $(14.8 ; 21.1)$ & 24.9 & $(21.6 ; 28.4)$ & \\
Cat. 3 & 0.5 & $(0.2 ; 3.0)$ & 0.2 & $(0.0 ; 0.7)$ & 0.02 \\
Deaths rate (\%) & 7.8 & $(5.6 ; 8.8)$ & 5.0 & $(3.2 ; 6.3)$ & 0.14 \\
Readmissions rate (\%) & 2.8 & $(2.4 ; 3.3)$ & 3.7 & $(2.6 ; 6.6)$ & 0.53 \\
Nurse work load (h) & 39.9 & $(38.8 ; 41.4)$ & 39.9 & $(38.7 ; 40.3)$ & 0.44 \\
Provided & 39.4 & $(37.5 ; 43.4)$ & 38.7 & $(36.1 ; 41.3)$ & 0.45 \\
Requested & 1.7 & $(0.9 ; 4.0)$ & 1.7 & $(0.9 ; 3.9)$ & $<0.001$ \\
ICU length of stay (days) & 21 & $(12 ; 34)$ & 29 & $(21 ; 40)$ & \\
SAPS II score & & & & & \\
\hline
\end{tabular}

Fig. 1 Time distribution of monthly readmission rate (solid bars, left-hand scale) as compared with total admissions numbers (open squares, righthand scale) before and after the intervention (vertical line)

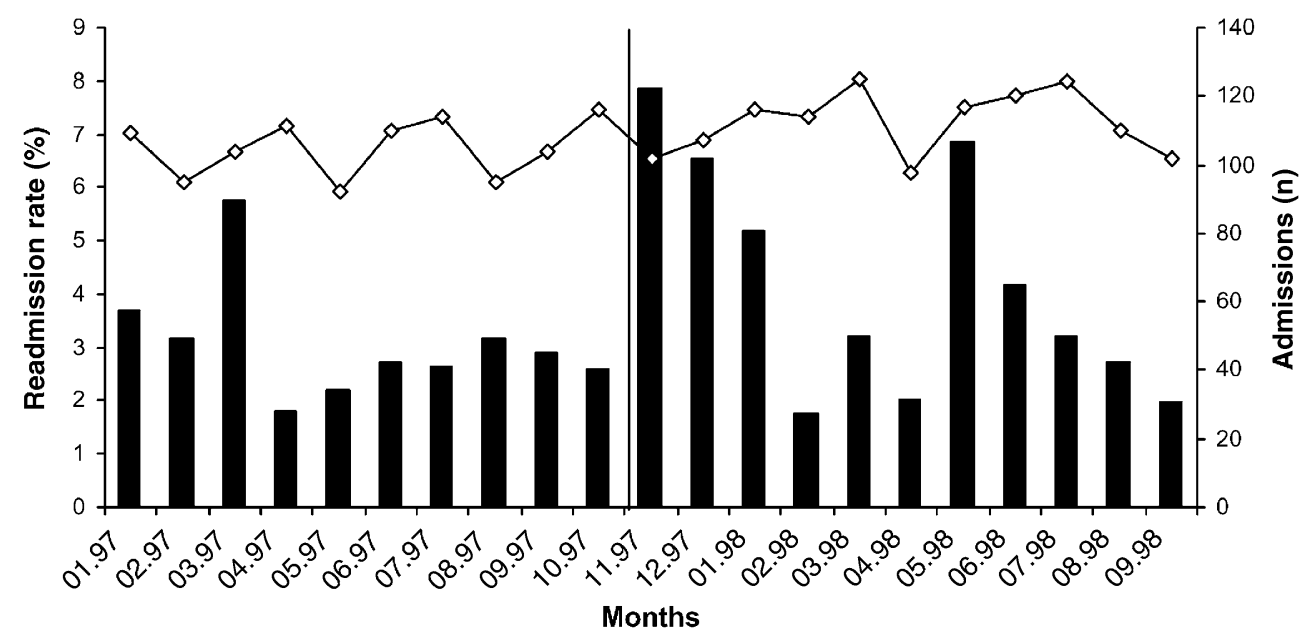

(Spearman $r=0.10$ vs 0.30 ). The increase in activity translated into an increase in ICU occupancy rate from a median of $69.1 \%$ to $79.7 \%$, and no decrease in LOS. Nurse workload did not change because staff was reduced by four full-time employees after bed closure. Patients' severity distribution as measured in days of care by SSMI categories was similar in the two periods, but physiologic scores as assessed with SAPS II score, significantly increased $(P<0.001)$. The global rate of deaths significantly decreased $(P=0.02)$. These findings contrasted with an observed significant increase $(P=0.05)$ in the number of readmissions (Fig. 1) but not in the readmission rate.
Activity in upstream units

Results of indicators collected in the recovery room before and after bed closure are displayed in Table 2. Surgical activity did not decrease on average after ICU bed closure, and the distribution of destinations of patients leaving the recovery room did not change. The case-mix of patients admitted to the recovery room, as measured by ASA score, did not change. The same was true for the other indicators.

Activity in downstream units

Results of available indicators before and after bed closure are displayed in Table 3. Activity in all intermediate care units did not significantly change, although 
Table 2 Comparison of patient orientation and anesthesiological risk categories in the recovery room before and after the monthly distributions) ICU bed closure (median of

\begin{tabular}{|c|c|c|c|c|c|}
\hline & Before & (Quartiles) & After & (Quartiles) & $\begin{array}{l}\text { Statistical } \\
\text { significance }\end{array}$ \\
\hline \multicolumn{6}{|l|}{ Patient destination (\%) } \\
\hline Ward & 89.8 & $(87.8 ; 91.4)$ & 86.3 & $(82.0 ; 89.8)$ & 0.728 \\
\hline Intermediate care unit & 5.9 & $(4.3 ; 9.0)$ & 7.5 & $(5.9 ; 10.0)$ & \\
\hline Intensive care unit & 0.2 & $(0.0 ; 0.4)$ & 0.1 & $(0.0 ; 0.6)$ & \\
\hline Other & 4.0 & $(2.0 ; 4.6)$ & 6.1 & $(4.4 ; 8.9)$ & \\
\hline \multicolumn{6}{|l|}{ ASA score $(\%)$} \\
\hline ASA 1 & 34.3 & $(31.9 ; 37.1)$ & 33.1 & $(31.6 ; 35.3)$ & 0.960 \\
\hline ASA 2 & 46.1 & $(43.8 ; 47.1)$ & 47.7 & $(44.3 ; 49.5)$ & \\
\hline ASA 3 & 18.6 & $(16.6 ; 19.9)$ & 18.2 & $(17.3 ; 20.2)$ & \\
\hline ASA 4 & 1.8 & $(1.1 ; 2.1)$ & 1.3 & $(1.0 ; 1.6)$ & \\
\hline ASA 5 & 0.0 & $(0.0 ; 0.1)$ & 0.0 & $(0.0 ; 0.1)$ & \\
\hline
\end{tabular}

Table 3 Comparison of activity and work load in intermediate care units and medical ICU before and after the bed closure (median of monthly distributions)

\begin{tabular}{lrlrll}
\hline & Before & (Quartiles) & After & (Quartiles) & $\begin{array}{l}\text { Statistical } \\
\text { significance }\end{array}$ \\
\hline Occupancy rate $(\%)$ & & & & \\
Cardiosurgery & 80.0 & $(76.3 ; 82.5)$ & 84.3 & $(77.8 ; 87.3)$ & 0.44 \\
Surgery & 69.7 & $(67.4 ; 79.8)$ & 71.7 & $(66.9 ; 80.1)$ & 0.53 \\
Neurosurgery & 81.0 & $(76.7 ; 87.6)$ & 88.5 & $(83.3 ; 91.4)$ & 0.14 \\
ENT surgery & 63.7 & $(60.2 ; 68.4)$ & 63.0 & $(50.9 ; 76.3)$ & 0.97 \\
Orthopedic surgery & 47.4 & $(38.7 ; 54.5)$ & 45.5 & $(39.4 ; 56.1)$ & 0.92 \\
Medical ICU & 87.2 & $(78.1 ; 98.1)$ & 89.1 & $(86.0 ; 90.6)$ & 0.74 \\
Work load (h) & & & & & \\
Provided & & & & \\
Cardiosurgery & 7.6 & $(7.5 ; 7.7)$ & 7.4 & $(7.3 ; 7.6)$ & 0.31 \\
Surgery & 15.2 & $(15.1 ; 15.4)$ & 14.6 & $(14.2 ; 15.1)$ & 0.06 \\
Neurosurgery & 7.0 & $(6.8 ; 7.1)$ & 7.2 & $(7.0 ; 7.4)$ & 0.08 \\
ENT surgery & 5.0 & $(4.8 ; 5.6)$ & 5.6 & $(5.0 ; 6.2)$ & 0.17 \\
ORTHopedic surgery & 3.5 & $(3.3 ; 3.7)$ & 3.5 & $(3.4 ; 3.6)$ & 0.85 \\
Medical ICU & 31.0 & $(29.5 ; 31.5)$ & 30.0 & $(29.7 ; 30.5)$ & 0.53 \\
Requested & & & & \\
Cardiosurgery & 7.9 & $(7.6 ; 8.0)$ & 7.9 & $(7.4 ; 8.3)$ & 0.48 \\
Surgery & 12.3 & $(12.0 ; 13.8)$ & 12.6 & $(11.4 ; 13.6)$ & 0.97 \\
Neurosurgery & 6.1 & $(5.8 ; 6.6)$ & 7.0 & $(6.7 ; 7.1)$ & 0.04 \\
ENT surgery & 5.2 & $(4.5 ; 5.5)$ & 4.9 & $(4.0 ; 5.6)$ & 0.74 \\
ORTHopedic surgery & 3.3 & $(2.9 ; 3.7)$ & 3.2 & $(2.9 ; 3.6)$ & 0.80 \\
Medical ICU & 31.0 & $(28.0 ; 37.8)$ & 31.4 & $(30.7 ; 33.2)$ & 0.80 \\
P & & & & \\
\hline
\end{tabular}

nursing workload significantly increased in neurosurgery. No impact could be noticed on mortality and costs in downstream units.

\section{Discussion}

This study demonstrated that a decrease of $6 \%$ in the surgical ICU bed capacity did not have a measurable impact upstream or downstream from it. Despite its high occupancy rate, already noticeable before bed closure, the ICU was able to accept, after the intervention, more patients who were significantly more severely affected $(P<0.001)$ at the expense of an increase in the rate of emergency admissions $(P=0.02)$, indicating that the planning of elective admissions was no longer possible, despite no change in the number of elective surgical operations. On the other hand, the rate of readmissions also increased, although not significantly. This pattern was observed not only on a short-term basis, reflecting adaptation to the new system, but it persisted on a midterm basis, indicating that patient pathways were no longer predictable.

Outcome indicators are often considered as the ultimate goal for assessing quality of care. However, this is true only when outcome can be measured immediately after the process of care, before other factors play a role. A systematic review of published studies about readmissions to ICUs did not find consistent data to support its use as a measure of quality of care [14]. Three main factors are linked with ICU readmissions [15]: disease progression, post-operative care requirement, and inadequate follow-up care on general wards. As readmissions are not correlated with severity-adjusted mortality, ICU LOS, or hospital LOS, this indicator must capture other components of care, and should be used in conjunction with other measures of hospital performance [16]. However, from a quality assurance perspective, erratic pat- 
terns recorded persistently after an intervention should dictate reversal to the initial situation before an accident happens.

Our findings could also suggest that efficiency was not optimal prior to bed closure or that total bed number was excessive. Both the occupancy rates and the distribution of patient's severity as assessed by SSMI classification do not support this claim. Other alternative explanations, such as a modification in processes of care, available technology, physician coverage, structure or staffing of other hospital units, did not occur. The observation periods were long enough to ensure that the seasonal variations in admission rates recently described [17], but not observed in our study, could not play a role.

ICU resources have always been limited compared with existing demand, and rationing admission is an everyday occurrence. As a consequence, patients treated during bed shortage are often more severely ill at admission, sicker at discharge, and have a shorter stay. This does not influence ICU or after-discharge death rates, and ICU readmission rate [18]. ICU bed closure can be safely carried out only under a number of conditions. First, there must be a pool of patients admitted for discretionary reasons (category III in SSMI classification), who can be moved to other wards: in a medical ICU, bed shortage caused by a lack of nurses led to a reduction of patients admitted primarily for monitoring, and earlier transfers out of the ICU [19]. Alternatively, intensive monitoring must be available in other wards: opening a high dependency unit resulted in a decrease of ICU bed occupancy rate by these kinds of patients from $21.6 \%$ to $11.2 \%$ [20]. Third, the interface between intensive and intermediate care units must be precisely defined, in particular admission and discharge criteria.

These conditions were not met at our institution: no discretionary pool of patients was available as only $1 \%$ of them were classified in category III. Intensive monitoring was not carried out in the recovery room and downstream units, as the proportion of transfers to normal ward or intermediate care units did not change. Finally, admission and discharge criteria for the interface between intensive and intermediate care units did exist at our institution, and were not modified. An in-depth analysis and consideration of these conditions could have avoided trying this experiment and facing its consequences.

To our knowledge, very few studies have addressed the impact bed closures in one unit have on other functionally-related units in a hospital. Besides the impact of the opening of a high dependency unit described above [20], a single study described the impact on critical care utilization of closure of an intermediate care unit [21]: it found that non-emergency ICU admissions significantly increased, while physiologic severity scores, such as APACHE II or TISS score, and nursing workload significantly decreased. At the same time, ICU LOS tended to decrease, and total hospital LOS significantly decreased. ICU LOS did not change because the increase in the number of short-stay patients with low disease severity was offset by the necessity of keeping patients in a more sever condition longer in the ICU, as step-down facilities were lacking. The interesting conclusion of this study was that it promoted reestablishment of the intermediate care unit.

Our study also has limitations: it involved only one center, and its surgical ICU, in one health care system, with a follow-up limited to 10 months. Data about patients refused access to ICU or prematurely discharged, and their impact on other wards or waiting lists, as well as on postponed or cancelled operations, were not available. Similarly, although we found no significant repercussion on the functioning of the medical ICU, impact on more qualitative aspects, such as adequacy of admission, was not measured. Therefore, our findings could be invalid in other settings.

It is well known that health care systems are incredibly flexible and can adapt to multiple external constraints. Physicians are responsible for efficient allocation of scarce resources [22] in order to reach technical efficiency in their wards [23]. Therefore, performance monitoring is essential, and must be applied not only to the involved unit, but also to upstream and downstream units, as multiple interactions exist between them, so that the modification of one factor in one of them can have an impact on the others. The lack of responsiveness of most commonly-used indicators to monitor hospital activity and patient outcome is more disturbing, as stretching capacities beyond limits can lead to threats to quality of care and manifest itself in various ways: infections [24] and mortality [25] rates increase when nurses' workload increases. Ultimately, nurses resign, leading to closing additional beds. Thus, the high flexibility of health care structures is both an advantage when delivering care to patients is at stake, and an inconvenience when performance monitoring is involved.

In conclusion, our observational study showed that a significant decrease in bed capacity of a university ICU, treating a high proportion of patients with organ failure requiring active supportive treatment, was followed by adaptative mechanisms, which individually had little impact on commonly-used indicators either in the ICU or upstream and downstream from it. In this context, the necessity of using multiple indicators of structure, processes, and outcomes to monitor such a managerial decision cannot be emphasized strongly enough. 


\section{References}

1. Gaynor M, Anderson GF (1995) Uncertain demand, the structure of hospital costs, and the cost of empty hospital beds. J Health Economics 14:291-317

2. Mackay M (2001) Practical experience with bed occupancy management and planning systems: an Australian view. Health Care Manag Sci 4:47-56

3. Bagust A, Place M, Posnett JW (1999) Dynamics of bed use in accommodating emergency admissions: stochastic simulation model. BMJ 319:155-158

4. Tu JV, Jagal SB, Naylor CD (1995) Multicenter validation of risk index of mortality, intensive care unit stay, and overall hospital length of stay after cardiac surgery. Circulation 91:677684

5. Gallivan S, Utley M, Treasure T, Valencia O (2002) Booked inpatient admissions and hospital capacity: mathematical modelling study. BMJ 324:280-282

6. Norris C, Jacobs P, Rapoport J, Hamilton S (1995) ICU and non-ICU cost per day. Can J Anaesth 42:192-196

7. Singer M, Myers S, Hall G, Cohen SL, Armstrong RF (1994) The cost of intensive care: a comparison on one unit between 1988 and 1991. Intensive Care Med 20:542-549

8. Rubenfeld GD, Angus DC, Pinsky MR, Curtis JR, Connors AF, Bernard GR, and the members of the Outcomes Research Workshop (1999) Outcomes research in critical care; results of the American Thoracic Society Critical Care Assembly Workshop on Outcomes Research. Am J Respir Crit Care Med 160:358-367
9. Le Gall JR, Lemeshow S, Saulnier F (1993) A new simplified acute physiology score (SAPS II) based on a European/North American multicenter study. JAMA 270:2957-2963

10. Owens WD, Felts JA, Spitznagel EL (1978) ASA physical status classification: a study of consistency of ratings. Anesthesiology 49:239-243

11. Tilquin C (1977) Un cadre conceptuel et méthodologique pour les classifications de malades selon leurs besoins en soins infirmiers. In: Coblentz AM, Walter JR (eds) Systems science in health care. Taylor \& Francis, London, pp 289-296

12. Rothen HU, Küng V, Ryser DH, Zurcher R, Regli B (1999) Validation of "nine equivalents of nursing manpower use score" on an independent data sample. Intensive Care Med 25:606611

13. Donabedian A (1988) The quality of care: how can it be assessed? JAMA 260:1743-1748

14. Rosenberg AL, Watts CM (2000) Patients readmitted to ICUs: a systematic review of risk factors and outcomes. Chest 118:492-502

15. Russel S (1999) Reducing readmissions to the intensive care unit. Heart Lung 28:365-372

16. Cooper GS, Sirio CA, Rotondi AJ, Shepardson LB, Rosenthal GE (1999) Are readmissions to the intensive care unit a useful measure of hospital performance? Med Care 37:399-408
17. Garfield M, Ridley S, Kong A, Burns A, Blunt M, Gunning K (2001) Seasonal variations in admission rates to intensive care units. Anaesthesia 56:1136-1140

18. Strauss MJ, LoGerfo JP, Yeltatzie JA, Temkin N, Hudson LD (1986) Rationing of intensive care unit services: an everyday occurrence. JAMA 255:1143-1146

19. Singer DE, Carr PL, Mulley AG, Thibault GE (1983) Rationing intensive care: physician response to a resource shortage. N Engl J Med 309:1155-1160

20. Fox AJ, Owen-Smith O, Spiers P (1999)

The immediate impact of opening an adult high dependency unit on intensive care unit occupancy. Anaesthesia 54:280-283

21. Byrick RJ, Mazer CD, Caskennette GM (1993) Closure of an intermediate care unit: impact on critical care utilization. Chest 104:876-881

22. Snider GL (1994) Allocation of intensive care; the physician's role. Am J Respir Crit Care Med 150:575-580

23. Puig-Junoy J (1998) Technical efficiency in the clinical management of critically ill patients. Health Econ 7:263-277

24. Vicca AF (1999) Nursing staff workload as a determinant of methicillinresistant Staphylococcus aureus spread in an adult intensive therapy unit. J Hosp Infect 43:109-113

25. Tarnow-Mordi WO, Hau C, Waedren A, Shearer AJ (2000) Hospital mortality in relation to staff workload: a 4-year study in an adult intensive-care unit. Lancet 356:185-189 